\title{
Resident Plug-In Electric Vehicle Charging Modeling and Scheduling Mechanism in the Smart Grid
}

\author{
Peng Han, Jinkuan Wang, Yinghua Han, and Yan Li \\ School of Information Science and Engineering, Northeastern University, Shenyang 110819, China \\ Correspondence should be addressed to Jinkuan Wang; wjk@mail.neuq.edu.cn
}

Received 18 October 2013; Accepted 10 December 2013; Published 2 January 2014

Academic Editor: Hui Zhang

Copyright ( 2014 Peng Han et al. This is an open access article distributed under the Creative Commons Attribution License, which permits unrestricted use, distribution, and reproduction in any medium, provided the original work is properly cited.

\begin{abstract}
With the development of smart grid and the increase of global resident Plug-In Electric Vehicle (PEV) market in the near future, the interaction between limited distribution grid capacity and uncontrollable PEV charging loads can lead to violations of local grid restrictions. And the proper model charging scheduling mechanism is the key to assess and satisfy various resident charging requirements and help in optimizing utility utilization. In this paper, the distribution grid profile model with PEV charging power is firstly constructed for the purpose of studying resident PEV charging impact on the distribution grid. To better reflect the actual impact of PEVs, we use real data on driving behaviors, vehicle characteristics, and electricity loads to generate our model. Furthermore, an improved queuing-theory-based scheduling mechanism is proposed, the distribution grid communication structure and the algorithm are illustrated, and computer simulations are demonstrated to verify their performance. The results show that the proposed scheduling mechanism will enhance the distribution grid flexibility to meet various charging requirements while maximizing the grid capacity.
\end{abstract}

\section{Introduction}

The smart grid represents a new paradigm in electrical power distribution and management. Incorporating advanced twoway communications and distributed computing capabilities, smart grid is emerging power grids that enhance control, efficiency, reliability, and safety. The resident Plug-In Electric Vehicle (PEV) is a motor vehicle that can be recharged by plugging in the house electricity source [1]. With zero greenhouse gas emission and a readily available energy source, PEV offers a promising alternative for residents as a new mean of transportation. The National Electromobility Development Plan, proposed by the German Federal Government in August 2009, predicts one million electric vehicles on German roads by 2020 [2]. And current estimations state that only by 2015 there would be more than 3 million electric vehicles on roads all around the world [3].

While this is a huge step forward for electric vehicles and emissions reduction, it will also cause a huge demand for electricity. Considering that the PEV batteries are sizeable and uncoordinated loads, the large-scale adoption of PEVs will significantly increase the power grid load especially in the peak load hours. And the coincidence between peaks of PEV charging load and non-PEV load will require massive investments in generation, transmission, and distribution [4, 5]. Moreover, since PEV adoption is initially expected to cluster in residential communities where the demand for PEVs is strongest [6], those PEVs will increase the potential for negative distribution system impacts, and the charging power may overload transformers and sap much needed distribution capacity, causing severe grid fluctuations and blackouts while degrading power grid efficiency [7].

On the other side, due to the PEVs' high battery capacities and high charging powers to store massive electricity at offpeak hours, they may serve as ideal actors for demand side management (DSM) and can maximize the yield of electricity supplied by renewable sources such as PV panels [8]. And the development of smart grid and AMI techniques enables utilities in many countries to adopt various price strategies or regulations, for example, time-of-use (TOU) tariff, to shift the PEVs charging requests [9]. However, the increasing penetration of resident PEVs brings various quality-of-service 
TABLE 1: PEV types and key parameters.

\begin{tabular}{|c|c|c|c|c|c|}
\hline \multicolumn{6}{|c|}{ Key parameters } \\
\hline PEV types ${ }^{a}$ & Prototypes & Battery capacity & Charge power & Max range & Market share \\
\hline Auto & Nissan LEAF & $24 \mathrm{kWh}$ & $6.6 \mathrm{~kW}$ & $73 \mathrm{~mL}$ & $49.9 \%$ \\
\hline SUV & AMP and Jeep Cherokee & $37.6 \mathrm{kWh}$ & $6.5 \mathrm{~kW}$ & $80 \mathrm{~mL}$ & $19.4 \%$ \\
\hline Pick-up & Smith Newton & $80 \mathrm{kWh}$ & $8 \mathrm{~kW}$ & $50 \mathrm{~mL}$ & $18.2 \%$ \\
\hline Truck & Smith Edison & $36 \mathrm{kWh}$ & $6 \mathrm{~kW}$ & $60 \mathrm{~mL}$ & $8.2 \%$ \\
\hline
\end{tabular}

${ }^{a} 2009$ National Household Travel Survey.

(QoS) requirements [10]. Moreover, if it goes without the proper charging scheduling management, the large charging activities that have been shifted to the night by price strategies will also bring night high-peak hours to the grid [11].

Considering all the facts above, in order to maintain the reliability of the power system, the appropriate model for evaluating and predicting PEV charging activity impact toward smart grid must be designed, and a proper PEV charging scheduling mechanism is critical for maintaining the grid safety and PEV charging efficiency. Several recent works currently are undergoing the effort to optimize the PEV charging activity. Asad et al. [12] adopted sensor web service to manage the charging activity, but the algorithm considers few parameters and is not practical. Callaway and Hiskens proposed a hysteresis-based control strategy in [13] to charge PEV intermittently, but that strategy will cause obvious oscillations due to charging synchrony. Taheri and Entriken proposed a clustering algorithm in [14] to consider future demands of vehicles in a fleet, and in their algorithm each vehicle's charging schedule is instantly determined as it connects to the grid; therefore, the scheduling process is not dynamic and lacks flexibility. Baek et al. in [15] put forward a queuing model for PEV charging systems; however, the proposed $\mathrm{M} / \mathrm{M} / \mathrm{Inf}$ model does not quite match the real PEV charging scenario in the smart grid.

Although a number of papers in this field have established the benefits of optimizing PEV charging activities, the PEV charging model in the smart grid has not been practically and clearly demonstrated; therefore, the real-time impact of PEVs on the distribution grid has yet to be fully explored. Moreover, considering the limited computational capabilities of smart grid terminals in the residential area, a relatively low complexity scheduling mechanism is preferred. Therefore, this paper proposed a resident PEV charging model based on the driving data from National Household Travel Survey (NHTS), 2009 [16]. The real-time grid profile data of the utility are also imported into this model to get the impact of charging activity. Based on the model this paper illustrates the communication structure of the distribution grid, and an improved queuing-theory-based scheduling algorithm is proposed, which would work to coordinate PEVs charging activity with the consideration of both distribution grid safety and the various PEV charging requirements. The simulation shows that the proposed mechanism can improve the ability of the distribution grid to better respond to the resident PEV charging demand while improving the reliability of the grid system.

The rest of this paper is organized as follows. Section 2 illustrates the communication structure of the smart grid with the adoption of PEVs, analyses the resident PEV charging activity, and proposes the model in the distribution grid. The charging activity impact of the PEV is demonstrated with the proposed model. Section 3 analyses scheduling using queuing theory and proposes the improved scheduling mechanism. The simulation is presented and discussed in Section 4 to inspect its effectiveness; then the paper concludes in Section 5.

\section{PEV Charging Activity Analysis and Modeling}

2.1. Key Parameters Analysis of PEV Charging Activity. Considering the fact that resident PEVs will be a major smart grid application [17], the key parameters that affect the power grid are the PEV types, resident driving habits, the distribution grid load profile, and PEV market.

(1) PEV Types. PEV type determines the vehicle's charging power $P_{c}$ and battery capacity $C$. Based on the 2009 NHTS database, the resident vehicles can mainly be classified by the following four types: auto, sport utility vehicle (SUV), pickup, and truck. To model the impact of PEV charging activities, we selected four PEV prototypes, respectively, for each type, and their key parameters are shown in Table 1. Nissan LEAF [18] is the latest version of pure electric autos in the market, and the electric Cherokee [19] developed by AMP and Jeep is an ideal substitution for ordinary SUV, while the Newton Pickup and Edison Truck produced by Smith [20] have been widely used in US transportation business.

(2) Resident Driving Habits. Driving habits of the drivers influence the charging start time $T_{s}$ and duration $T_{l}$ directly, where $T_{s}$ is mainly determined by the home-arrival time, and $T_{l}$ depends on the daily driving range together with the battery capacity and charging power. Given the assumption that the resident driving habits stay unchanged and PEVs will get recharged immediately when arriving home, the twodimensional conditional probability distribution diagram between daily range and home-arrival time on weekdays and weekends is constructed based on the statistical data in NHTS 2009. The two distribution diagrams are illustrated in Figures 1 and 2. As can be observed, the majority of PEVs should be charged between 15:00-20:00 on the weekdays and 18:0023:00 on the weekends, and the daily ranges are mostly within 50 miles.

(3) Distribution Grid Load Profile. The PEV charging load will superimpose directly onto the real-time distribution 


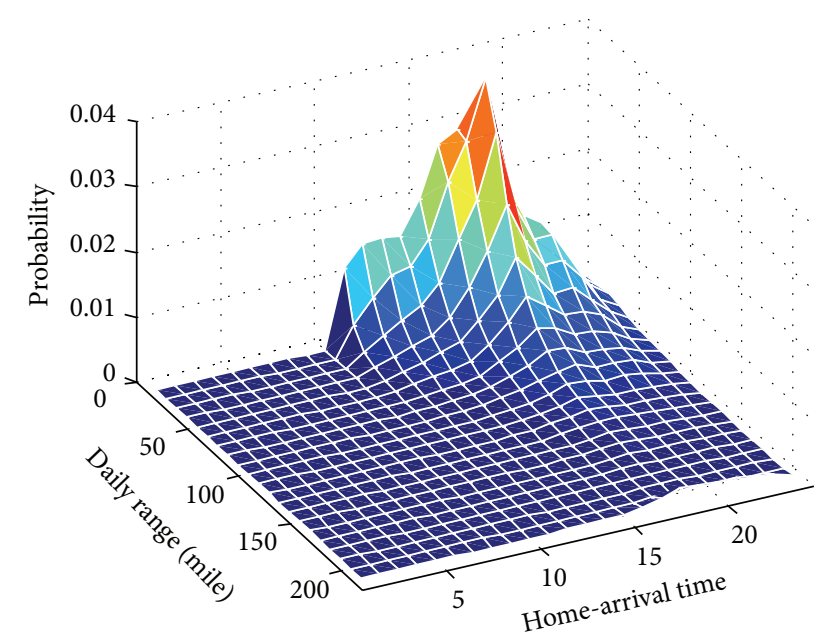

FIGURE 1: Conditional probability distribution diagram in weekdays.

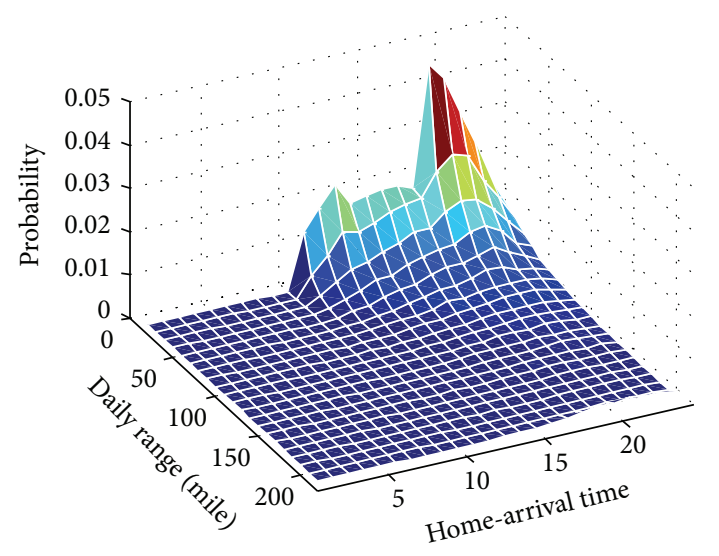

FIGURE 2: Conditional probability distribution diagram in weekends.

grid load curve $P_{G}(t)$. And therefore the available power for PEV charging activities depends on the original grid load profile $P_{\text {load }}$ and the distribution grid capacity $P_{\text {all }}$, that is, the maximum power allowance. In this paper, the electricity grid load profile consults the real load of the Electric Reliability Council of Texas (ERCOT) [21]. ERCOT is an isolated electrical system operator that manages the flow of electric power to 23 million customers in Texas; this paper calculates the average power consumption on the hour and interpolates the hourly data into continuous power profile $P_{G}(t)$. Considering that the large-scale PEV data is not presently available, the paper reasonably assumes a residential community with oneten-thousandth of Texas population, and all houses in this community are equipped with the smart meters. Provided that the residential consumption ratio $\theta$ of Texas electricity is $37.6 \%$ [22], the residential community original grid load profile $P_{\text {load }}$ can be calculated from the function:

$$
P_{\text {load }}=\frac{P_{G}(t) \theta e}{E}, \quad 0 \leq t<24,
$$

where $e$ is the population of the proposed residential community, which is supposed to be 2300 as one-ten-thousand of the population of Texas E. Given that the ERCOT capacity is $83000 \mathrm{MW}$, the community distribution grid capacity $P_{\text {all }}$ is assumed to be $3000 \mathrm{~kW}$.

(4) PEV Market. The number of PEVs $N$ on a certain area depends on its population $e$, vehicles ownership ratio $R$, and the PEV penetration ratio $\mu$. Due to the NHTS 2009, the vehicles ownership ratio $R$ is $74 \%$. Therefore, given the 2300-population residential community, the vehicle amount is about 1702 .

2.2. PEV Charging Activity Modeling. Considering the charging activity from the $i$ th $\mathrm{PEV}$, the charging time duration $T_{l i}$ can be calculated from the function:

$$
T_{l i}=\frac{l_{i} C_{i}}{K_{i} P_{c i} \rho},
$$

where $l_{i}$ is the driving miles, $C_{i}$ is the battery capacity, $K_{i}$ is the maximum range, $P_{c i}$ is the charging power, and $\rho$ is the charging efficiency. Provided the PEV charging start time, the charging end time $T_{e i}$ can be calculated by the function:

$$
T_{e i}=T_{s i}+T_{l i} .
$$

Without the loss of generality, the arrival time of the PEV in this paper is regarded to be a nonstationary Poisson process [23], and the probability distribution function of $K$ newarrival charging PEVs in each hour is the function:

$$
P(X=k)=\frac{e^{-\lambda_{t}} \lambda_{t}^{k}}{k !}
$$

where $\lambda_{t}$ is the expectation of hourly new-arrival charging PEVs, and it can be calculated from the function:

$$
\lambda_{t}=\lambda_{f} e R \mu,
$$

where $\lambda_{f}$ is the piecewise function of PEV charging time hourly fractions on weekdays and weekends, which can be derived from Figures 1 and 2. Therefore, considering the proposed community with all the above parameters, the real power load on the distribution grid when adopted with PEVs $P_{\text {real }}$ can be expressed in the function:

$$
\begin{array}{r}
P_{\text {real }}=P_{\text {load }}+\sum_{i=1}^{R} P_{c i}\left[u\left(t-T_{e i}\right)-u\left(t-T_{s i}\right)\right], \\
0 \leq P_{\text {real }} \leq P_{\text {all }},
\end{array}
$$

where $u(t)$ is the step function.

2.3. PEV Charging Impact Simulation. Based on the proposed model, the simulation of PEV charging impact to the electricity grid is demonstrated in Figure 3. The PEV penetration ratio is set to be 0.4 . The thin and thick dashed red wave represents the power of original distribution grid load $P_{\text {load }}$ in summer weekdays and weekends, and the dotted line in 


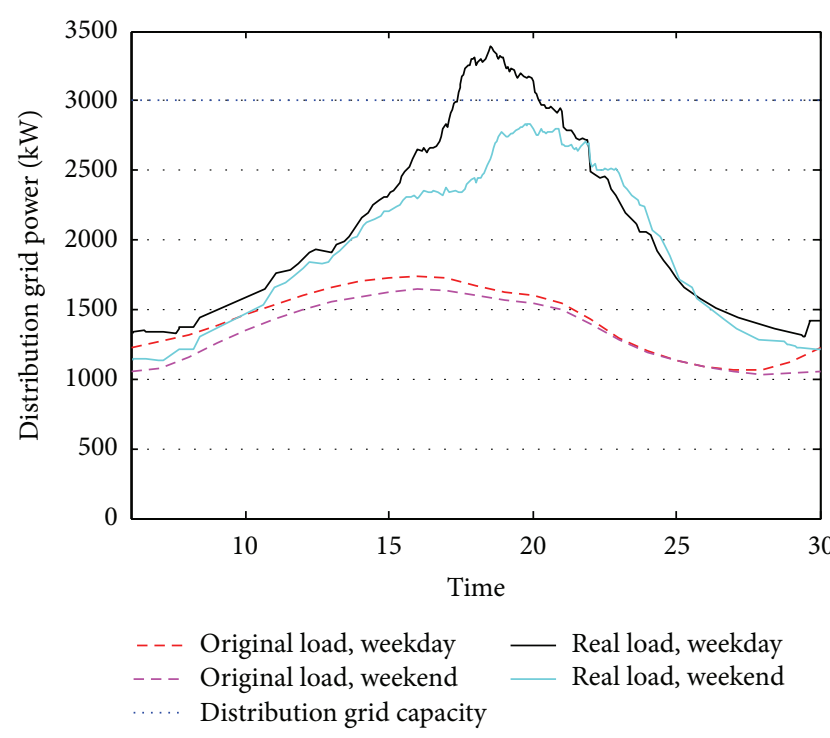

FIGURE 3: PEV charging activity impact without the proper management.

$3000 \mathrm{~kW}$ is the distribution grid capacity. The thin and thick solid lines denote the distribution grid load $P_{\text {real }}$ which includes the addup of PEV charging power.

As is indicated in Figure 3, the PEV charging activity without proper management conducts a severe grid fluctuation. The power profile caused by mass PEV charging activity significantly reshapes the original grid load power profile and enlarges the peak time as well as the peak value. Due to the coincidence of the night charging activities, the charging load around 20:00 p.m. rises to the distribution grid capacity, which might cause critical accidents in reality. However, the load after midnight is nearly unchanged, which denotes that the power generation in the night is not well utilized. In order to maximize the grid utilization to support the future growth of PEV market without investing heavily in upgrading existing grid capacity, the PEV charging activity scheduling mechanism should be developed.

\section{PEV Charging Activity Scheduling Mechanism}

3.1. Distribution Grid Communication Structure. In this paper, it is assumed that most of the PEVs owners in the proposed community have been interested in participating in utility scheduling by charging or discharging their PEVs. And therefore a proper communication structure based on the smart grid is necessary to enable the utility's scheduling instruction and the PEV owners' timely response. Considering a PEV-adopted distribution grid system, we can identify three main communication network components between resident houses and distribution station: wide area network, neighborhood area network, and home area network.

As is demonstrated in Figure 4, in order to achieve the scheduling, each resident PEV should be monitored and powered by a smart meter in the house of the PEV owner.
The smart meter is an intelligent device that manages the power supply of the home area network (HAN) in the smart grid, and the control of the house owner or utility will be performed by the predeployed smart sockets. Smart meters in a certain area, for example, a residential community, can form a neighborhood area network (NAN) through wireless mesh networks, power line communication (PLC), or Ethernet and handle the last-mile connectivity from the smart meters located on the edge of the power grid to the grid data collectors. Each data collector coordinates a large number of houses and manages the communication between a group of residents and an electric distribution utility, collecting smart meter data and transferring the utility instruction and information through wide area network (WAN), and the public wired (solid line) and wireless (dashed line) communication networks with a ubiquitous reach are critical elements of successful WAN communication.

3.2. Queuing-Theory-Based Scheduling Algorithm. There are currently no standard algorithms on regulating PEV charging activity in the smart grid. Although several proposed sophisticated management mechanisms relying on real-time high-speed communications could allow distribution circuits to run at nearly full capacity, the cost-effectiveness would then compromise due to the sophisticated mechanism, and complex negotiation between the resident and the utility may result in an unsuccessful outcome [24]. Considering the realistic scenario of the smart grid in the near future, scheduling algorithm based on relatively low computing complexity should be more appropriate. They can be easily deployed in smart meters and other smart devices; meanwhile their communications require relatively few network resources to manage hundreds of PEV charging activities in a certain area. Queuing theory is adopted in a widespread manner for analyzing and optimizing stochastic problems, as they provide a range of benefits in scheduling the available resource to maximize the need of consumers with low computing complexity, which is suitable for the low-cost and low-power smart sockets and can be well applied in smart meters.

There are many different queuing regulations for different circumstances, which follow a different scheduling algorithm and have their own advantages and disadvantages. firstcome first-served (FCFS) is the basic scheduling algorithm for PEV charging in many grids and charging stations. The main process of scheduling mechanism with FCFS algorithm is illustrated in Figure 5(a). If the grid is in peak hours when new PEV arrives and there is no available power for charging, the PEV will be suspended and regulated by the FCFS queuing regulation which places all PEVs in a queue and charges them in the order that they arrive in. And as soon as the available distribution grid power is sufficient for the headmost PEV in the queue, the FCFS algorithm will automatically trigger the charging process of that vehicle.

Due to its low complexity and fairness of the service, the FCFS scheduling has been considered as a possible strategy in scheduling the PEV charging activity in the smart grid [15]. However, this method cannot distinguish PEV charging requests with urgency. In order to satisfy the various 


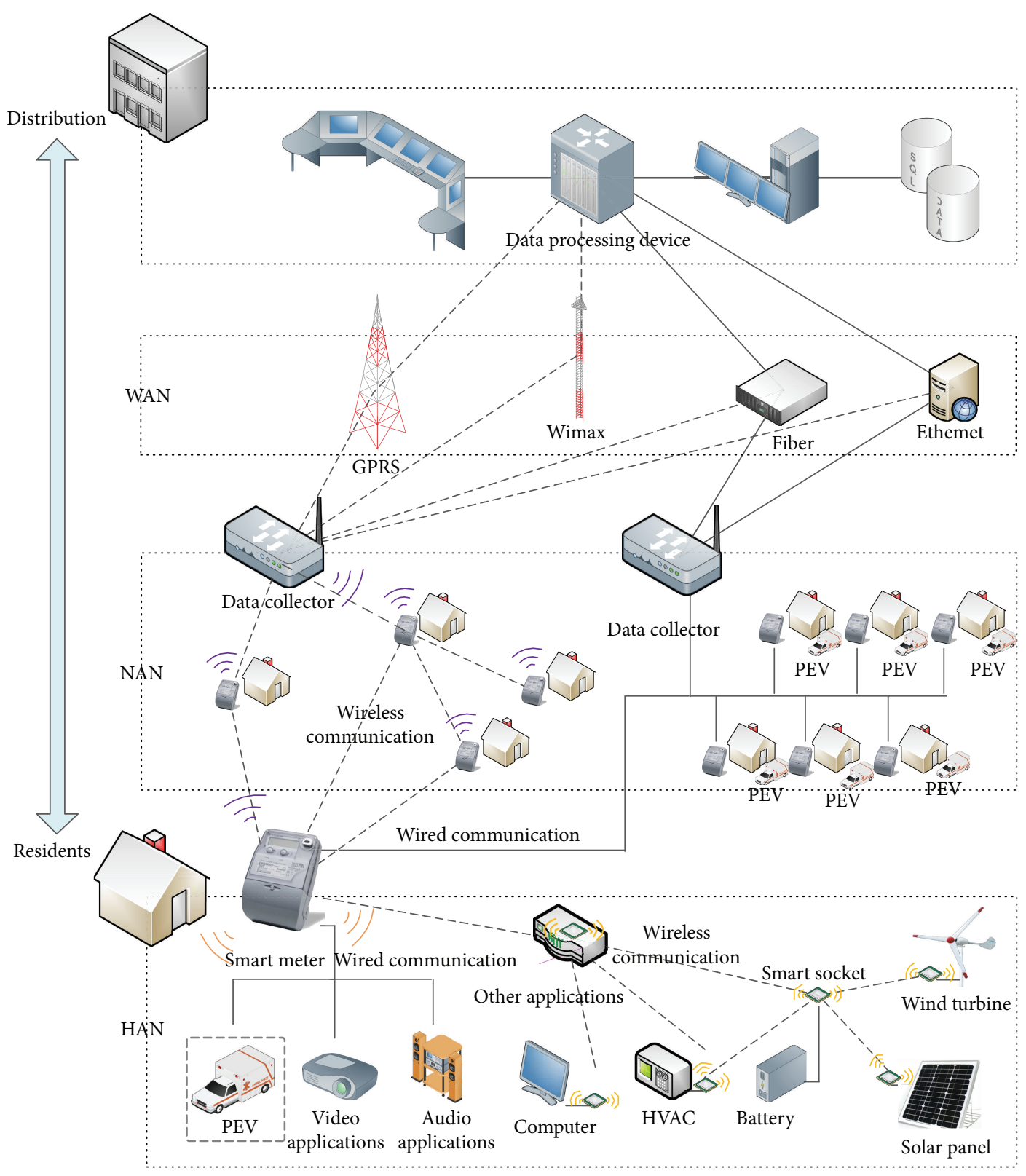

FIgURE 4: Distribution grid communication structure.

charging requirements brought by the increasing penetration of PEVs, this paper proposes an improved queuing-theorybased dynamic scheduling algorithm that assigns each PEV with different priority levels, with which the schedule queue can be considered as a soft real-time system comprising mainly aperiodic tasks $[25,26]$. Therefore, the PEV priority is assigned based on the slack time of each process, which is defined in the function:

$$
T_{k i}=T_{d i}-T_{s i}-T_{l i}
$$

where $T_{d i}$ is the allowable latest charging finish time and can be calculated by the function:

$$
T_{d i}=T_{p i}-T_{s i}
$$

where $T_{p i}$ is the resident-preset charging duration time through smart meters, and it reflects the resident charging urgency. The main process of dynamic scheduling algorithm is illustrated in Figure 5(b).

\section{Computer Simulation}

4.1. Simulation Platform and Configuration. The computer simulation is demonstrated based on the proposed model in order to verify the performance of the proposed scheduling mechanism. Considering that the PEV charging events of a certain area form a typical discrete event process, the ExtendSim software is adopted as the simulation platform to establish the proposed model. ExtendSim is advanced simulation software with powerful constructs and unlimited hierarchical 


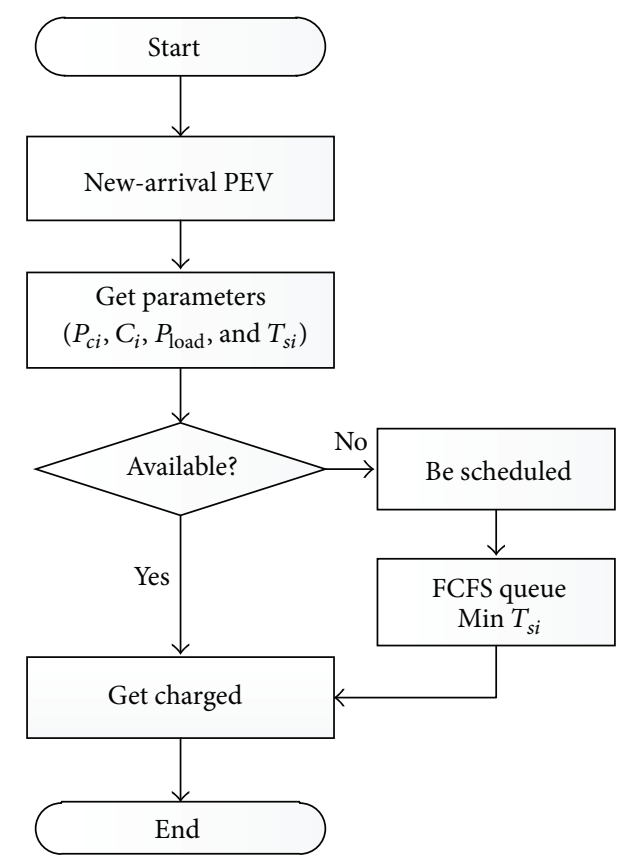

(a)

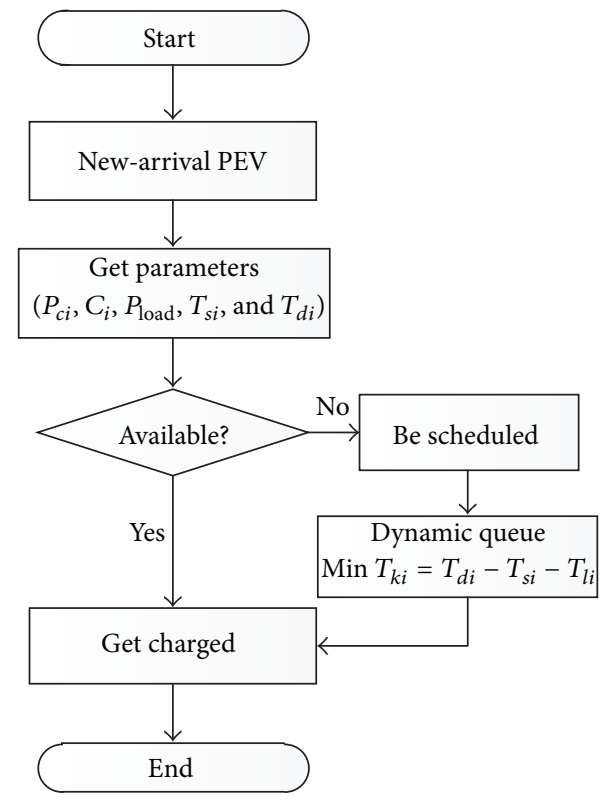

(b)

FIGURE 5: Main process of scheduling mechanisms.

TABLE 2: Key parameters of the simulation.

\begin{tabular}{lccccc}
\hline Population & Vehicle owner & PEV penetration ratio & Charging efficiency & Power capacity & Power threshold \\
\hline 2300 & 1702 & 0.4 & 0.833 & $3000 \mathrm{~kW}$ & $2400 \mathrm{~kW}$ \\
\hline
\end{tabular}

structure for modeling complex discrete event processes [27], which is especially suitable for the modeling the dynamic PEV charging queuing process and multiple scheduling algorithms. Meanwhile, the features of the ExtendSim also make the constructed model flexible and extensible for more complex situations in the future study.

In order to compare the performance of the proposed algorithms as well as the FCFS, the main simulation parameters including the resident population, PEV types and specification, and the driving habit of the proposed residential community keep the same as those in Section 2, where the key parameters are listed in Table 2 . And in order to represent the charging PEVs with high, middle, and low urgency, $T_{p i}$ in the simulation is preset to be $1 \mathrm{~h}, 3 \mathrm{~h}$, and $6 \mathrm{~h}$ with the probability of $0.3,0.3$, and 0.4 .

With the simulation parameters added and modules connected in the ExtendSim platform, the main simulation structure of the proposed model is shown in Figure 6, where the scheduling algorithms are programmed in function modules marked with a black block in the figure.

4.2. Simulation Result and Discussion. A series of computer simulations are conducted based on the above conditions. Figure 7 shows $P_{\text {real }}$ with the FCFS algorithm and the proposed algorithm, in which group 1 and group 2 denote the $P_{\text {real }}$ profiles in weekdays and weekends.
Compared with $P_{\text {real }}$ in Figure 3, electricity grid and the two PEV charging scheduling algorithms both enjoy a stable charging load in charging peak hours. And the performance of the two algorithms is similar to each other. The reshaped electricity grid load power profiles are safely limited under the $2400 \mathrm{~kW}$ threshold. Moreover, the power valley after midnight is largely filled up, and therefore the PAR is reduced. In order to examine the proposed scheduling algorithm in satisfying the PEV owners' requirements, the comparable simulation between the FCFS scheduling and the proposed scheduling is illustrated in Figure 8, in which the thin and thick solid lines denote the FCFS and proposed algorithms performance in weekdays and dashed lines are their performance in weekends.

Compared to the traditional FCFS scheduling, the proposed scheduling algorithm gives the PEV with smaller slack time $T_{k i}$ higher priorities; therefore, it has relatively higher on-time ratio, which helps to insure the charging of urgent PEV owners. The key parameters in the simulations have been listed in Table 3. It can be observed that the proposed algorithm has similar average queue length, maximum queue length, and average wait time. However, to ensure residents charging urgency requirements, it regulates residents with low urgency requirements with relatively longer wait time. Therefore, the maximum wait time of the proposed algorithm in weekdays and weekends is larger than FCFS, but the whole on-time rate is guaranteed. Moreover, in the simulation 


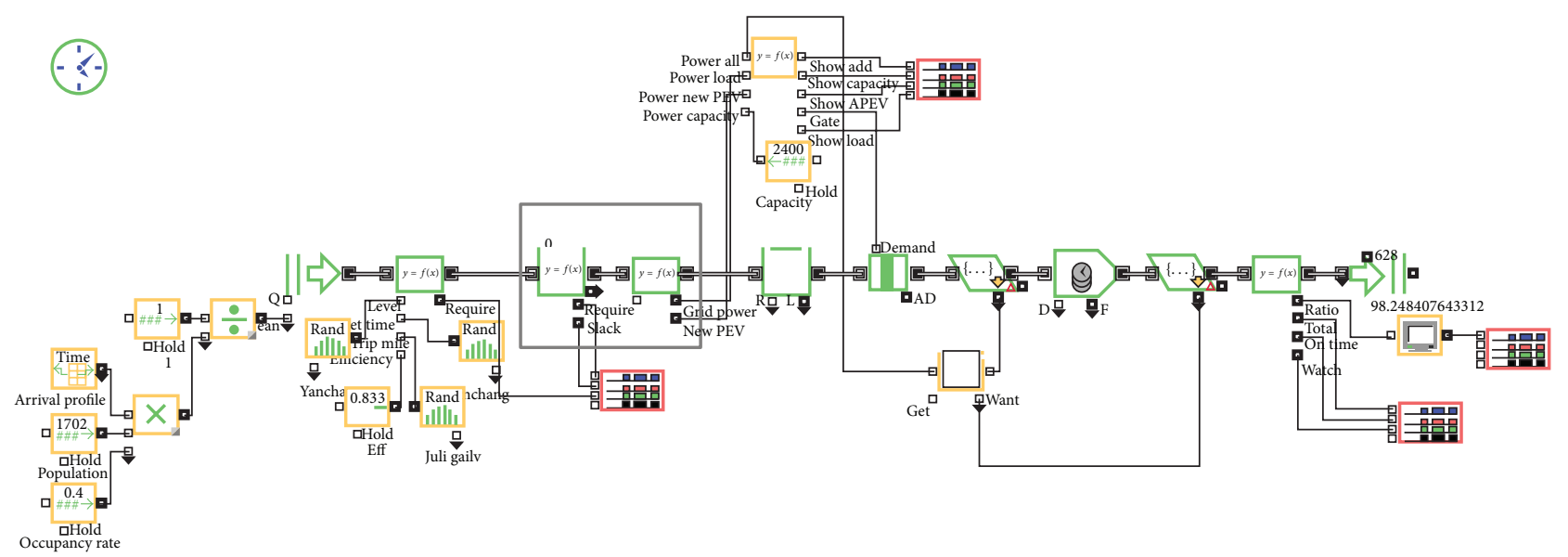

FIGURE 6: Main simulation structure of the proposed model.

TABLE 3: FCFS and proposed algorithm performance analysis.

\begin{tabular}{|c|c|c|c|c|c|}
\hline \multirow[b]{2}{*}{ Algorithm } & \multicolumn{4}{|c|}{ Key parameters } & \multirow[b]{2}{*}{ Max. wait } \\
\hline & On-time rate & Ave. queue & Max. queue & Ave. wait & \\
\hline FCFS weekdays & 64.39 & 47.85 & 239 & $2.14 \mathrm{~h}$ & $3.85 \mathrm{~h}$ \\
\hline Proposed weekdays & 98.24 & 47.87 & 244 & $2.15 \mathrm{~h}$ & $5.88 \mathrm{~h}$ \\
\hline FCFS weekends & 85.82 & 14.35 & 108 & $0.65 \mathrm{~h}$ & $1.49 \mathrm{~h}$ \\
\hline Proposed weekends & 100.00 & 13.29 & 98 & $0.60 \mathrm{~h}$ & $3.30 \mathrm{~h}$ \\
\hline
\end{tabular}



FIGURE 7: PEV charging impact with FCFS and proposed algorithm.

most of night-coming PEVs are served before 06:00 a.m. the next day, which denotes the proposed charging algorithm's efficiency in maintaining the electricity grid safety as well as satisfying the PEV owner charging requirements.

In practice, the proposed scheduling can be adopted with dynamic electricity prices; therefore, the idle PEV owners are encouraged to preset a larger $T_{d i}$ for an electricity bill discount, while urgent PEV owners can get charged in

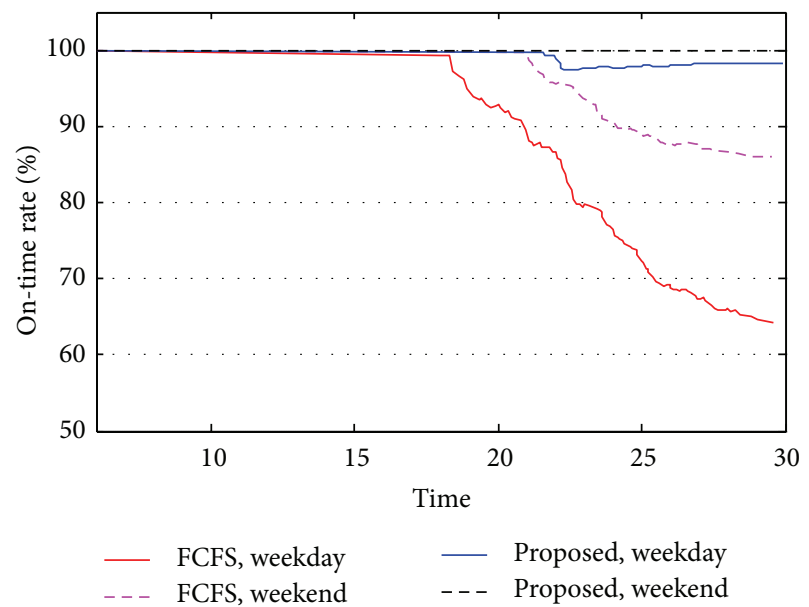

FIGURE 8: On-time ratio comparison.

advance at a cost of high bills. It should be noted that due to the fact that PEV is owned mostly by residential consumers, in this analysis it is assumed that the proposed charging mechanism is associated with home charging for simplicity. Nevertheless, in case it becomes necessary to account for the charging at public locations, the proposed scheduling and dispatch methods would still be properly applied as well.

\section{Conclusions}

The paper proposes a new PEV charging activity model and develops a queuing-theory-based mechanism to schedule the 
PEV charging in order to maximize the utilization of excess distribution circuit capacity while keeping the probability of a distribution grid load profile negligible. The proposed scheduling algorithm shows preferable performance in alleviating the charging impact. And by adopting the mechanism, the limited available power in high-peak load times is well utilized, the significant peak load is reduced, and the various charging requirements are largely satisfied. Due to its simplicity and flexibility, the model can be easily facilitated for grid system adopting vast amounts of PEVs.

\section{Conflict of Interests}

The authors declare that there is no conflict of interests regarding the publication of this paper.

\section{Acknowledgments}

This work is supported by the National Natural Science Foundation of Hebei, China, under Grant no. 201202073. The authors would sincerely show their appreciation to the editor and the anonymous reviewers for their invaluable comments.

\section{References}

[1] E. Ungar and K. Fell, "Plug in, turn on, and load up," IEEE Power and Energy Magazine, vol. 8, no. 3, pp. 30-35, 2010.

[2] German Federal Government, National Electromobility Development Plan Executive Summary, German Federal Government, Berlin, Germany, 2009.

[3] Pike Research, Investment in electric vehicle IT systems to total $\$ 5.1$ billion by 2015, http://www.pikeresearch.com/newsroom/smart-grid-investment-to-total-200-billion-worldwideby-2015.

[4] J. T. Salihi, "Energy requirements for electric cars and their impact on electric power generation and distribution systems," IEEE Transactions on Industry Applications, vol. 9, no. 5, pp. 516532, 1973.

[5] A. Y. Saber and G. K. Venayagamoorthy, "Plug-in vehicles and renewable energy sources for cost and emission reductions," IEEE Transactions on Industrial Electronics, vol. 58, no. 4, pp. 1229-1238, 2011.

[6] F. Ahmad, R. M. Hledik, A. Levy, and A. L. Madian, "Will smart prices induce smart charging of electric vehicles?" July 2011, http://www.smartgrid.gov/sites/default/files/doc/files/Will_Smart_Prices_Induce_Smart_Charging_Electric_Vehicles_201107. pdf.

[7] S. Ruthe, V. Schmutzler, and C. Rehtanz, "Study on V2G protocols against the background of demand side management," Ibis, vol. 11, pp. 33-44, 2011.

[8] W. Kempton and J. Tomić, "Vehicle-to-grid power fundamentals: calculating capacity and net revenue," Journal of Power Sources, vol. 144, no. 1, pp. 268-279, 2005.

[9] Y. Cao, S. Tang, C. Li et al., "An optimized EV charging model considering TOU price and SOC curve," IEEE Transactions on Smart Grid, vol. 3, no. 1, pp. 388-393, 2012.

[10] J. Gartner and C. Wheelock, "Electric vehicles?: 10 predictions for 2011," Tech. Rep., Pike Research, Boulder, Colo, USA, 2011.

[11] P. Fairley, "Speed bumps ahead for electric-vehicle charging," IEEE Spectrum, vol. 47, pp. 13-14, 2010.
[12] O. Asad, M. Erol-Kantarci, and H. Mouftah, "Sensor network web services for demand-side energy management applications in the smart grid," in Proceedings of the IEEE Consumer Communications and Networking Conference (CCNC '11), pp. 11761180, Las Vegas, Nev, USA, January 2011.

[13] D. S. Callaway and I. A. Hiskens, "Achieving controllability of electric loads," Proceedings of the IEEE, vol. 99, pp. 184-199, 2011.

[14] N. Taheri and R. Entriken, "A dynamic algorithm for facilitated charging of plug-in electric vehicles," Management Science, pp. $1-16,2011$.

[15] S. J. Baek, D. Kim, S.-J. Oh, and J.-A. Jun, "A queuing model with random interruptions for electric vehicle charging systems," in Proceedings of the IEEE International Conference on Consumer Electronics (ICCE '11), pp. 679-680, Las Vegas, Nev, USA, January 2011.

[16] US Department of Transportation, Summary of Travel Trends: 2009 National Household Travel Survey, Federal Highway Administration, Washington, DC, USA, 2012.

[17] J. Taylor, A. Maitra, M. Alexander, D. Brooks, and M. Duvall, "Evaluation of the impact of plug-in electric vehicle loading on distribution system operations," in Proceedings of the IEEE Power and Energy Society General Meeting (PES '09), pp. 26-30, Calgary, Canada, July 2009.

[18] Nissan Leaf Electric Car, http://www.nissanusa.com/leaf-electric-car/index.

[19] AMP to electrify Jeep Grand Cherokee, http://news.carjunky. com/alternative_fuel_vehicles/Amp_Electric_Jeep_Grand_Cherokee.shtml.

[20] Smith Electric Vehicles, http://www.smithelectric.com/smithvehicles/.

[21] FERC, Annual Electric Balancing Authority and Planning Area Report, http://www.ferc.gov/docs-filing/forms.asp\#714.

[22] The Energy Report of Texas, http://www.window.state.tx.us/ specialrpt/energy/.

[23] K. Turitsyn, N. Sinitsyn, S. Backhaus, and M. Chertkov, "Robust broadcast-communication control of electric vehicle charging," in Proceedings of the IEEE International Conference on Smart Grid Communication, pp. 4-6, Gaithersburg, Md, USA, 2010.

[24] M. Mültin, F. Allerding, and H. Schmeck, "Integration of electric vehicles in smart homes-an ICT-based solution for V2G scenarios," in Proceedings of the IEEE PES Conference on Innovative Smart Grid Technologies, pp. 16-20, Washington, DC, USA, 2011.

[25] M. Hwang, D. Choi, and P. Kim, "Least slack time rate first: an efficient scheduling algorithm for pervasive computing environment," Journal of Universal Computer Science, vol. 17, no. 6, pp. 912-925, 2011.

[26] A. K. Mok, Fundamental design problems of distributed systems for the hard-real-time environment [Ph.D. thesis], Massachusetts Institute of Technology, Cambridge, Mass, USA, 1983.

[27] ExtendSim Advantages, http://www.extendsim.com/prods_advantage.html. 


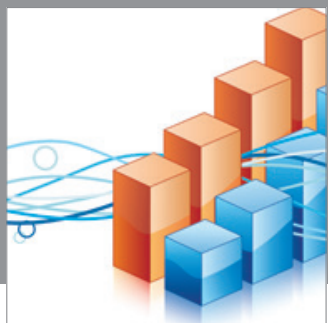

Advances in

Operations Research

mansans

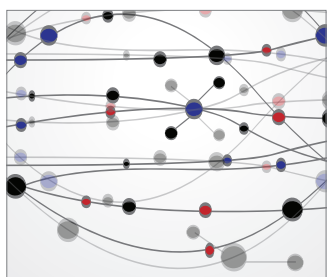

The Scientific World Journal
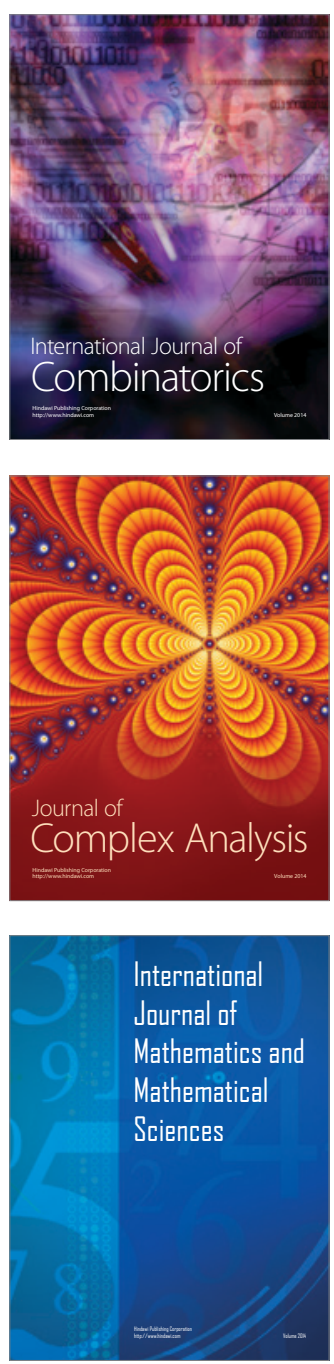
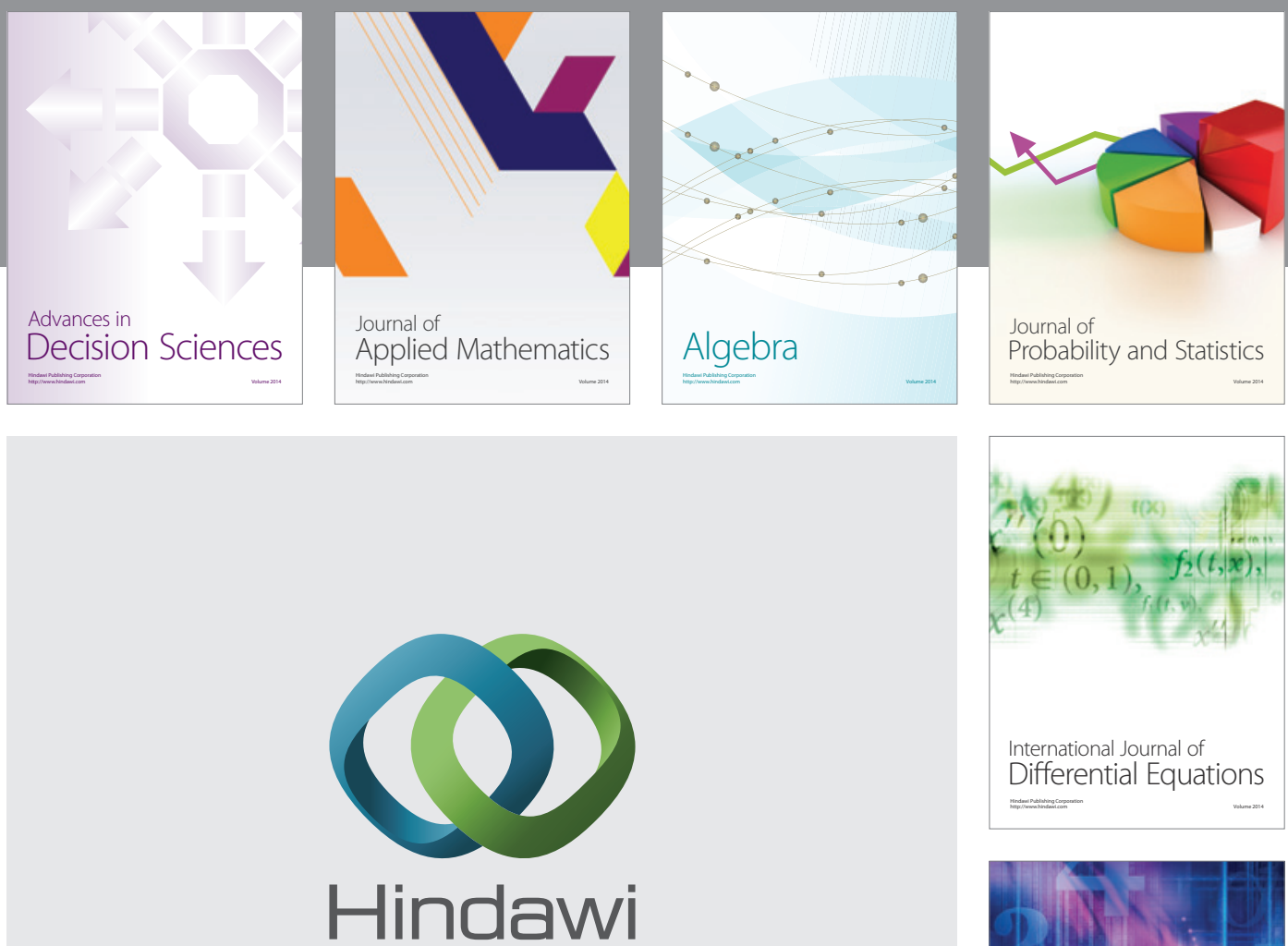

Submit your manuscripts at http://www.hindawi.com
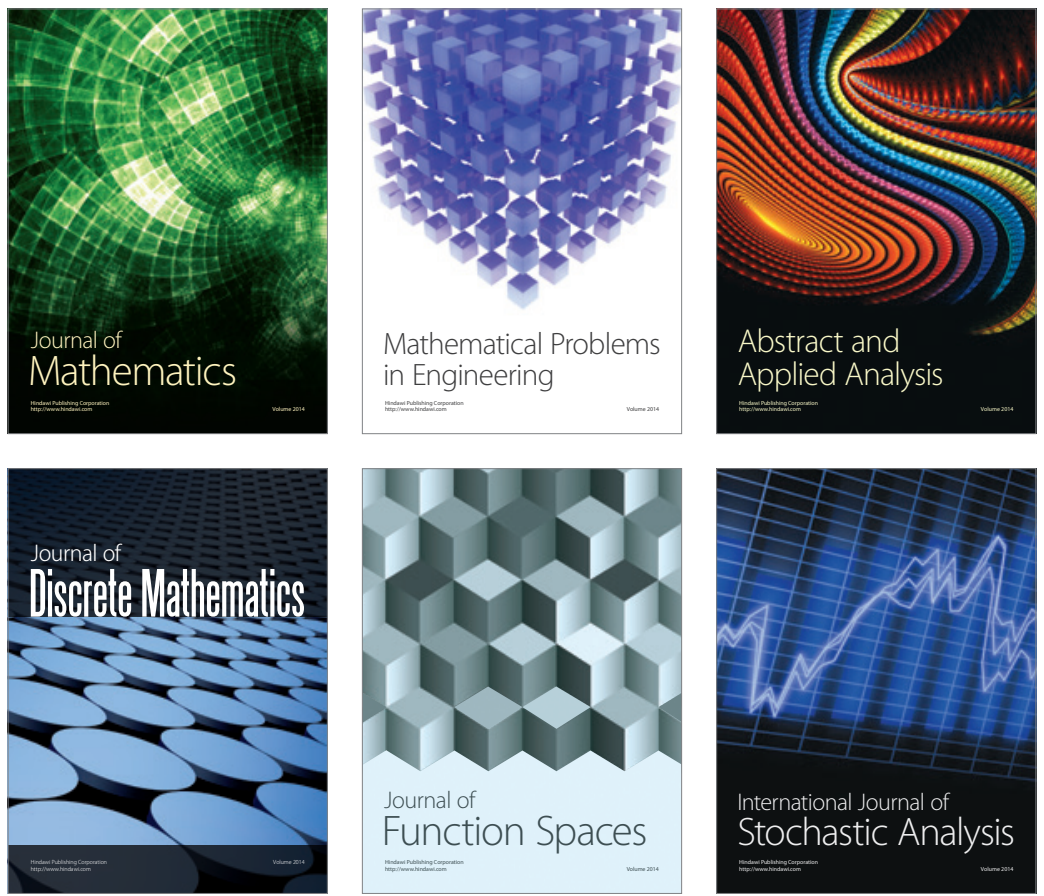

Journal of

Function Spaces

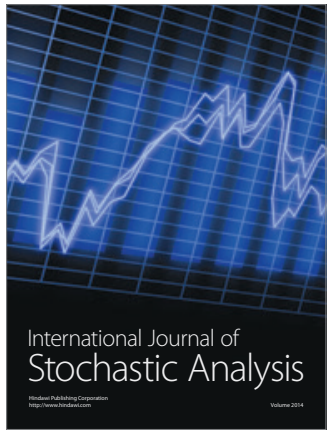

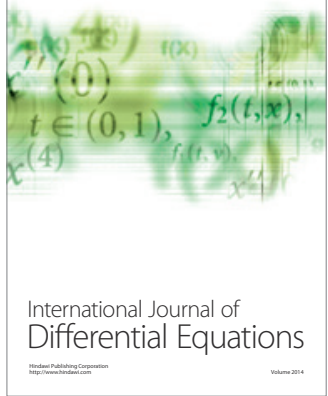
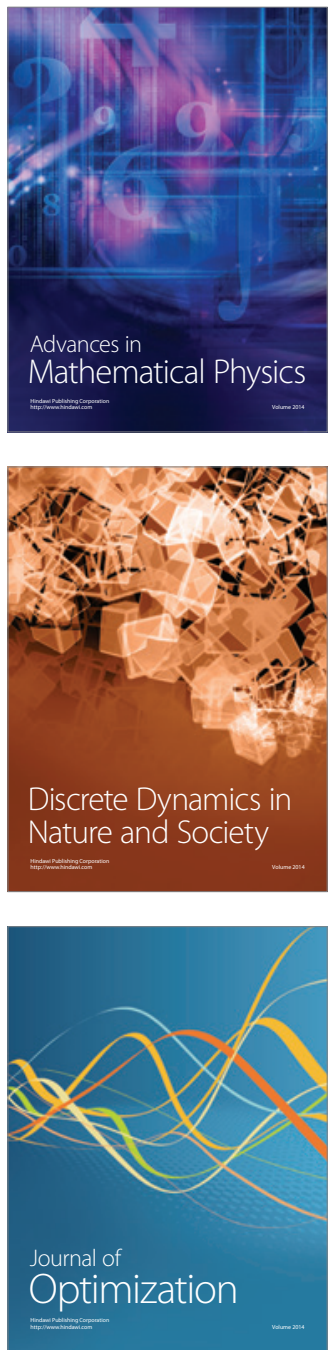